\title{
KIf8 regulates left-right asymmetric patterning through modulation of Kupffer's vesicle morphogenesis and spaw expression
}

Che-Yi Lin ${ }^{1,6 \dagger}{ }^{1}$ Ming-Yuan Tsai ${ }^{2,6 \dagger}{ }^{+}$Yu-Hsiu Liu ${ }^{3}$, Yu-Fen Lu ${ }^{4}$, Yi-Chung Chen ${ }^{4}$, Yun-Ren Lai ${ }^{1}$, Hsin-Chi Liao ${ }^{1}$, Huang-Wei Lien ${ }^{5}$, Chung-Hsiang Yang ${ }^{5}$, Chang-Jen Huang ${ }^{5}$ and Sheng-Ping L. Hwang ${ }^{1,3,4^{*}}$

\begin{abstract}
Background: Although vertebrates are bilaterally symmetric organisms, their internal organs are distributed asymmetrically along a left-right axis. Disruption of left-right axis asymmetric patterning often occurs in human genetic disorders. In zebrafish embryos, Kupffer's vesicle, like the mouse node, breaks symmetry by inducing asymmetric expression of the Nodal-related gene, spaw, in the left lateral plate mesoderm (LPM). Spaw then stimulates transcription of itself and downstream genes, including Ift1, Ift2, and pitx2, specifically in the left side of the diencephalon, heart and LPM. This developmental step is essential to establish subsequent asymmetric organ positioning. In this study, we evaluated the role of krüppel-like factor 8 ( $k$ lf8) in regulating left-right asymmetric patterning in zebrafish embryos.

Methods: Zebrafish klf8 expression was disrupted by both morpholino antisense oligomer-mediated knockdown and a CRISPR-Cas9 system. Whole-mount in situ hybridization was conducted to evaluate gene expression patterns of Nodal signalling components and the positions of heart and visceral organs. Dorsal forerunner cell number was evaluated in $\mathrm{Tg}($ sox 17:gfp) embryos and the length and number of cilia in Kupffer's vesicle were analyzed by immunocytochemistry using an acetylated tubulin antibody.

Results: Heart jogging, looping and visceral organ positioning were all defective in zebrafish klf8 morphants. At the 18$22 \mathrm{~s}$ stages, klf8 morphants showed reduced expression of genes encoding Nodal signalling components (spaw, Ift1, Ift2, and pitx2) in the left LPM, diencephalon, and heart. Co-injection of klf8 mRNA with klf8 morpholino partially rescued spaw expression. Furthermore, klf8 but not klf8 $\Delta f$ overexpressing embryos showed dysregulated bilateral expression of Nodal signalling components at late somite stages. At the 10s stage, klf8 morphants exhibited reductions in length and number of cilia in Kupffer's vesicle, while at 75\% epiboly, fewer dorsal forerunner cells were observed. Interestingly, klf8 mutant embryos, generated by a CRISPR-Cas9 system, showed bilateral spaw expression in the LPM at late somite stages. This observation may be partly attributed to compensatory upregulation of klf12b, because klf12b knockdown reduced the percentage of klf8 mutants exhibiting bilateral spaw expression.
\end{abstract}

Conclusions: Our results demonstrate that zebrafish KIf8 regulates left-right asymmetric patterning by modulating both Kupffer's vesicle morphogenesis and spaw expression in the left LPM.

Keywords: Zebrafish, Klf8, Spaw, L-R patterning, Kupffer's vesicle

\footnotetext{
* Correspondence: zoslh@gate.sinica.edu.tw

†'Equal contributors

${ }^{1}$ Department of Bioscience and Biotechnology, National Taiwan Ocean University, Keelung, Taiwan

${ }^{3}$ Department of Life Science, National Taiwan University, Taipei, Taiwan

Full list of author information is available at the end of the article
} 


\section{Background}

Despite the outward appearance of bilateral symmetry in vertebrates, internal organs exhibit substantial left-right asymmetry. In humans, genetic disorders that affect leftright asymmetric patterning may result in organ heterotaxy [1], complex congenital heart disease, and asplenia/ polysplenia [2]. In order to study the various processes that establish left-right asymmetry in a laboratory setting, several vertebrates, including mice and zebrafish, have been utilized. Largely based on these animal studies, the major developmental processes which establish asymmetry are known to include: symmetry-breaking in the node, the transfer of asymmetric Nodal expression from the node to the left lateral plate mesoderm (LPM), asymmetric expression of Nodal and downstream genes in the left LPM, and the completion of left-right asymmetric organ morphogenesis [3, 4].

Clockwise rotation of nodal cilia creates a directional nodal flow, which is responsible for the preferential activation of Nodal expression on the left side of the embryo [5]. Not surprisingly, mutations in genes involved in ciliogenesis [6] or its regulation [7, 8] have been found to disrupt normal left-right patterning. Leftward nodal flow generates an initial accumulation of NODAL protein on the left side of the embryo. Subsequently, selfenhancement and lateral-inhibition systems involving NODAL, LEFTY1 and LEFTY2 reinforce the asymmetric distribution and restrict Nodal gene expression to the left side of the organism [9].

Nodal signalling is initiated by the binding of NODAL to the ACTIVIN receptor and EGF-CFC co-receptor, which results in the formation of an intracellular regulatory complex. This complex consists of phosphorylated SMAD2, SMAD4 and FoxH1, and directly activates target gene transcription [10]. Left-side specific enhancers (ASEs) with FoxH1 binding motifs are present in the murine Nodal and Lefty2 genes [11]. Thus, NODAL amplifies its own expression in the left LPM via SMADs/FoxH1 interaction with the ASE. Simultaneously, NODAL induces Lefty2 expression, which inhibits low-level NODAL signalling, and thereby restricts Nodal expression to the left LPM. This asymmetric NODAL activation induces expression of Pitx2 in the left LPM, via its ASE. PITX2 is a homeodomain transcription factor implicated in left-right asymmetric organ morphogenesis [12], and loss of Pitx2 expression has been shown to affect the asymmetric distribution of internal organs in several vertebrates [13].

In zebrafish embryos, Kupffer's vesicle (KV) performs a similar role to the mouse node in initiating left-right asymmetric patterning [14]. KV is derived from dorsal forerunner cells (DFCs), which are formed via a Nodal signalling-dependent ingression of surface enveloping layer cells from the dorsal blastoderm margin. This ingression occurs at the blastula stage, when embryonic epiboly initiates [15]. DFCs then migrate toward the vegetal pole and organize into multiple rosette-like, epithelial structures at the end of gastrulation. These epithelial rosettes then merge into a single epithelial rosette and differentiate into the ciliated $\mathrm{KV}$, with the vesicle lumen arising from apical membrane expansion during early somite stages. Tilted cilia are positioned with the basal body at the posterior pole of DFCs, and these motile cilia are asymmetrically distributed along the anterior-posterior axis of KV. Furthermore, cilia asymmetry is established by the Rho kinase, Rock $2 b$, and is essential to generate an anti-clockwise swirling flow that commences asymmetric Nodal signalling [16-18]. In addition to rock $2 b$, deficiencies in several genes involved in the movement, formation or positioning of cilia, such as dnah9, ift88, and vangl2, have been shown to disrupt normal left-right patterning $[14,19,20]$.

Three nodal-related genes, namely ndr2 (cyc), ndr1 (sqt), and southpaw (spaw), have been identified in zebrafish [10]. Among these genes, spaw exhibits the earliest expression in the left-side of the LPM, and stimulation of its own transcription during somitogenesis shifts its expression domain from the posterior to the anterior left LPM [21, 22]. Furthermore, morpholino knockdown of spaw decreases expression of genes encoding Nodal signalling components, including spaw, lefty1 (lft1), lefty2 (lft2), and pitx2, in the left LPM [22], affecting the left-right asymmetric distribution of heart, pancreas, and diencephalon. Together, these studies demonstrate the essential role of Spaw, and underscore its relevance as a NODAL homolog in establishing leftright asymmetry of teleosts $[22,23]$.

Similar to mouse embryos, different repressors of the Nodal signalling pathway have been reported to modulate the induction or maintenance of asymmetric Nodal signalling in teleosts. At the 4-6 s stages, spaw is expressed bilaterally in $\mathrm{KV}$, while charon is expressed in a region adjacent to KV, where it antagonizes Spaw activity and contributes to biased spaw expression in the left LPM [24]. Furthermore, repression of Spaw activity in the right LPM or cardiac field by Lft1 or Lft 2 is also essential in the establishment of left-right patterning. Notably, lft 1 expression in the notochord is induced via binding of BMP4 with BMP receptor 1aa at the early segmentation stage, while lft 2 expression in the left cardiac field is activated by Spaw in the anterior LPM [25-27]. Despite this detailed knowledge about Spaw repressor proteins, it is still unknown whether asymmetric spaw expression in the left LPM can be regulated by transcription factors.

Krüppel-like factor 8 (KLF8) is a member of the KLF family of transcription factors [28, 29], and participates in a broad range of developmental processes. KLF proteins 
contain C-terminal zinc finger DNA binding motifs, and distinct N-terminal regulatory elements. KLF8, like KLF3 and KLF12, possesses a regulatory domain that interacts with C-terminal binding protein (CtBP) [30]. Interaction of KLF8 with the co-repressor CtBP inhibits embryonic Gamma-Globin gene expression [31, 32], a role confirmed in klf8-deficient mice [33]. KLF8 also functions as a mediator of focal adhesion kinase to activate cyclin D1 expression, modulating cell cycle progression [34]. Recently, we used morpholino knockdown and rescue experiments to show that zebrafish Klf8 has a novel function in cerebellar development. In this context, Klf8 modulates the expression of $p 53$ and met to maintain ptf1a-expressing neuronal progenitors, which are required for proper development of cerebellar Purkinje and granule cells [35]. In addition, we noted that $k l f 8$ morphants often exhibited a no-loop heart at $48 \mathrm{~h}$ post fertilization (hpf).

In this study, we demonstrate that zebrafish Klf8 plays an additional role in regulating left-right asymmetric patterning. Heart jogging, looping and visceral organ positioning were defective in klf8 morphants. At 18-22 s stages, expression levels of spaw, lft1, lft2, and pitx2 were decreased or eliminated in the left LPM, diencephalon, and heart of the majority of klf8 morphants. In contrast, $k l f 8$ overexpression resulted in bilateral expression of spaw and its downstream target genes in these tissues. Both dorsal forerunner cell number, and the length and number of cilia in $\mathrm{KV}$ were also affected in klf8 morphants. However, klf8 CRISPR-Cas mutant embryos showed bilateral spaw expression in the LPM, which may have been partly due to compensatory upregulation of klf12b.

\section{Methods}

\section{Ethics approval}

All animal procedures were approved by the Institutional Animal Care and Use Committee of Academia Sinica (Protocol ID: 15-12-918).

\section{Zebrafish maintenance and staging}

Adult AB zebrafish, $\operatorname{Tg}(\operatorname{sox} 17: g f p)^{s 870 /+}$ and klf8 mutants $\left(k l f 8^{d 25}, k l f 8^{i 17}\right)$, generated by a CRISPR-Cas9 system were maintained in high density, self-circulation systems (Aqua Blue), or $20 \mathrm{~L}$ aquaria supplied with filtered fresh water and aeration under a photo period of $14 \mathrm{~h}$ light and $10 \mathrm{~h}$ dark. Embryos were maintained at $28.5^{\circ} \mathrm{C}$, and morphological criteria were defined as described [36].

\section{Plasmid construction, morpholino and mRNA injection}

The full-length $k l f 8$ coding sequence or klf 8 lacking zinc finger domain $(k l f 8 \triangle z f)$ was cloned into the T7TS vector, and used as template to synthesize capped mRNA with the mMESSAGE mMACHINE T7 Kit (Ambion). Previously published morpholinos (MOs) were used [35], including two MOs that prevent Klf8 protein translation: $k_{l f} l-\mathrm{MO}^{\text {atg }}(2.2 \mathrm{ng})$ and $k l f 8-\mathrm{MO}^{\text {atg }}(1.9 \mathrm{ng})$, two MOs that prevent klf8 mRNA splicing: $\mathrm{MO}^{\mathrm{DO}}(0.73 \mathrm{ng})$ and $\mathrm{MO}^{\mathrm{AC}}(0.73 \mathrm{ng})$, and one control MO: klf8-4 mm MO1 (2.2 ng). An additional MO to prevent klf8 mRNA splicing: $\mathrm{MO}^{\mathrm{DO}}$ (0.73 ng; 5' - TGGGTCACATCCATCT CACCTGATC - $3^{\prime}$; targets the donor site of exon 3) was used. A 1.5-fold greater dosage of P53 $\mathrm{MO}^{\mathrm{sp}}$ [37] was injected, as compared to the co-injected klf8-MOs. To verify upregulation of $k l f 12 b$ in homozygous $k l f 8^{d 25}$ mutant F6 embryos, $k l f 12 b$ MO (5 or $10 \mathrm{ng}$ ) was used. $k l f 12 b \mathrm{MO}$ (5'-ATTCCGTCTAGCATTAACATCCTGT-3'), which is complementary to 20 bases of the coding region including the ATG start codon (underlined) and five bases of the $5^{\prime}$ untranslated region, was used. The indicated MO or mRNA was microinjected into one- or two-cell zygotes using a Nanoject II automatic injector (Drummond).

\section{Whole mount in situ hybridization, whole mount immunohistochemistry, and photography}

Whole mount in situ hybridization was performed on embryos treated with $0.003 \%$ phenylthiocarbamide, using digoxigenin-antisense RNA probes and alkaline phosphatase-conjugated anti-digoxigenin antibody. Various templates derived from pGEMT or pGEMTEasy vectors were linearized, and the following antisense RNA probes were generated (restriction site and promoter in parentheses): $b m p 2 b$ (BamHI/T7), charon (BamHI /Sp6), myl7 (NcoI /SP6), gata5 (SacII/ SP6), gata 6 (SalI/T7), lft1 (SalI/T7), lft2 (HindIII/ $\mathrm{SP} 6)$, ntl (Xhol/T7), oep (Ncol/SP6), pitx2c (EcoRI/ $\mathrm{T} 7$ ), and spaw (SpeI/T7). To produce the $d v r 1$ antisense RNA probe, PCR product that was generated using M13 forward and M13 reverse primers was used as a template and transcribed by T7 RNA polymerase.

To detect changes in KV cilia, one- or two-cell zygotes of $\operatorname{Tg}(\operatorname{sox} 17: g f p)$ were microinjected with different $k l f 8$ MOs. The 10s stage embryos were fixed in $4 \%$ paraformaldehyde for $2 \mathrm{~h}$ at room temperature (RT) and dehydrated in methanol at $-20{ }^{\circ} \mathrm{C}$. After dehydration, the embryos were permeabilized using acetone at $-20{ }^{\circ} \mathrm{C}$ and washed by Phosphate-buffered saline with tween 20 (PBST) followed by blocking in 5\% serum. The embryos were incubated with anti-acetylated tubulin antibody (1:250, Sigma-Aldrich) for $2 \mathrm{~h}$, at RT, followed by mouse Alexa Fluor 568 for $1 \mathrm{~h}$, at RT (1:250, Invitrogen).

To investigate DFC number alteration, one- or two-cell zygotes of $\operatorname{Tg}(\operatorname{sox} 17: g f p)$ were microinjected with different klf8-MOs. 75\% embryos were fixed in $4 \%$ paraformaldehyde at $4{ }^{\circ} \mathrm{C}$ overnight. After dehydration, the embryos were permeabilized using acetone at $-20{ }^{\circ} \mathrm{C}$ for $7 \mathrm{~min}$ and treated with $0.15 \mathrm{M}$ Tris- $\mathrm{HCl}, \mathrm{pH} 9.0$ at $70{ }^{\circ} \mathrm{C}$ for $15 \mathrm{~min}$. The embryos were washed with PBST followed by blocking 
in $1 \%$ blocking solution (Roche) at RT for $2 \mathrm{~h}$. The embryos were incubated with anti-GFP antibody (1: 200) at $4{ }^{\circ} \mathrm{C}$ overnight, followed by rabbit Alexa Fluor 488 (1:200, Invitrogen) incubation for $1 \mathrm{~h}$, at RT. Nuclei were then stained with Hoechst 33,341 (1:1000 in PBST, Invitrogen).

Brightfield embryo images were taken using an AxioCam HRC camera mounted on a Zeiss Imager M1 microscope. Fluorescent images were taken using a Leica TCS-SP5-MP confocal microscope.

\section{Generation of klf8 mutants using CRISPR-Cas9 system}

klf8 mutants were generated using a CRISPR-Cas9 system targeting exon 2. sgRNA was designed by ZGENE BIO BIOTECH INC. (Taipei, Taiwan) and DNA template was amplified from pZGB plasmids containing klf8 sgRNA. PCR was conducted using forward (5'-ACA CAGGAAACAGCTATGACCATG-3') and reverse (5'GATCCG CACCGACTCGGTGCCACTTT-3') primers, and klf8 sgRNA were synthesized using the MEGAshortscript T7 Transcription Kit (Ambion, Austin, TX, USA). $k l f 8$ sgRNA (86.3 pg) and capped nls-zCas9-nls mRNA (34.5 pg, Addgene) [38] were co-injected into one-cell zygotes. Genomic DNA was isolated from pools of 10 embryos at $24 \mathrm{hpf}$. PCR was conducted using forward (5'- ТСТTТСТАСТССТСССССААСТАA-3') and reverse (5'- CCACACCCCTTTCCAATAACTCTA-3') primers and amplified DNA was then digested with T7 endonuclease I to evaluate insertion and deletion efficiency. The rest of the embryos were reared to adulthood. Injected fish were designated as the F0 generation. To detect the DNA sequence alterations induced by klf 8 sgRNA, genomic DNA was isolated from clipped tail fin of adult F1 fish, and high resolution melt analysis was performed. PCR was conducted in a $20 \mu \mathrm{L}$ reaction comprising 8-12 ng genomic DNA, $3.5 \mathrm{mM} \mathrm{MgCl}_{2}, 1 \times$ Master Mix containing Taq DNA polymerase, dNTP mix and LightCycler 480 ResoLight dye, and 5 pmol each of forward (5' - ATCTCAGAACTCGGGTCACTTTTT-3') and reverse (5' - CCACCATACACTCCACCTCCTC-3') primers. The PCR conditions were $95^{\circ} \mathrm{C}$ for $300 \mathrm{~s}, 1$ cycle for preincubation, $95{ }^{\circ} \mathrm{C}$ for $10 \mathrm{~s}, 65{ }^{\circ} \mathrm{C}$ to $53{ }^{\circ} \mathrm{C}$ with a $0.5^{\circ} \mathrm{C}$ gradient decline for $10 \mathrm{~s}$, and $72{ }^{\circ} \mathrm{C}$ for $10 \mathrm{~s}$, for 70 cycles of amplification, and $95{ }^{\circ} \mathrm{C}$ for $60 \mathrm{~s}, 40{ }^{\circ} \mathrm{C}$ for $60 \mathrm{~s}, 65^{\circ} \mathrm{C}$ to $95{ }^{\circ} \mathrm{C}$ for $1 \mathrm{~s}$ melting interval for high resolution melting. DNA sequencing was conducted to confirm F1 adult fish with induced DNA sequence alterations. Two klf8 F1 mutants including $k l f 8^{d 25}$ with a 25 bp deletion and $k l f 8^{i 17}$ with a $17 \mathrm{bp}$ insertion in the exon $2 \mathrm{klf} 8$ sgRNA target site were crossed with wild type fish to produce the F2 generation. Subsequently, homozygous F4 $k l f 8^{d 25}$ and $k l f 8^{i 17}$ mutants were generated by intercrossing of respective heterozygous F3 mutants and maintained to the F5 generation.

\section{Reverse transcription PCR (RT-PCR) and reverse} transcription quantitative real-time PCR (RT-qPCR) RT-PCR was used to evaluate the efficacy of sp.-MOs. cDNA from $24 \mathrm{hpf}$, forward primer (5' - ATCAAGCC GGAGCCAGAGGAGGTG-3') and reverse primer (5'GCCGTCGGTGAAGTGCCAGGTG-3') were used. RTqPCR [39] was used to compare expression levels of klf3, $k l f 12 a$ or $k l f 12 b$ in wild type and homozygous F5 $k l f f^{d 25}$ and $k l f 8^{i 17}$ mutant embryos. cDNA was generated by a GoScript Reverse transcription system (Promega) using total RNA isolated from 10 to $12 \mathrm{~s}$ stage wild type or two homozygous $k l f 8^{d 25}$ and $k l f 8^{i 17}$ mutant embryos. RT-qPCR was conducted in a $10 \mu \mathrm{L}$ reaction containing $1 \times$ LightCycler 480 SYBR Green I Mix (Roche), respective primer pairs $(5 \mu \mathrm{g})$ and $1 / 10 \mathrm{cDNA}$ from wild type or mutant embryos. PCR conditions were $95{ }^{\circ} \mathrm{C}$ for $10 \mathrm{~min}$, then 55 cycles of, $95{ }^{\circ} \mathrm{C}$ for $10 \mathrm{~s}, 60{ }^{\circ} \mathrm{C}$ for $10 \mathrm{~s}$, and $72{ }^{\circ} \mathrm{C}$ for $10 \mathrm{~s}$, followed by a $4{ }^{\circ} \mathrm{C}$ pause. Primer pairs for $k l f 3$ were forward (5'-TATCCAAGTGGACATTACTGTGGG-3') and reverse (5'-CAGTGGGCAACACAGAACGGCAG-3'). Primer pairs for $k l f 12 a$ were forward (5'-GAGCGGGTCTC TTTCTGCCAGTG-3') and reverse (5'-CAATAAACCGTATGAGGGAAAGGC-3'). Primer pairs for $k l f 12 b$ were forward (5'-GGCAATCCCTGCTCCTCAGAAAC-3') and reverse (5' -CCACATCGTAGACTCCAAAATGCG-3').

\section{Quantification of cilia number and length and lumen of Kupffer's vesicles as well as DFC number}

The cilia length and number were quantified using LAS AF and MetaMorph software according to the following steps: (i) merge images with LAS AF for MetaMorph analysis; (ii) "Threshold Image" was set to demarcate cilia and KV cell locations, and the image was converted to grayscale; (iii) from the Arithmetic menu, "Logical AND" was selected, and KV cilia regions were defined; (iv) "Calibrate Distances" was set to define units of length $(\mu \mathrm{m})$; and $(v)$ the length and number of cilia were quantified by selecting "Integrated Morphometry Analysis" in the Measure menu. The area of KV lumen was quantified using ImageJ software as follows: (i) The merged grayscale images from MetaMorph were loaded in ImageJ; (ii) "Elliptical selections" was selected to demarcate cilia area; (iii) "Set scale" was selected to define units of area $\left(\mu \mathrm{m}^{2}\right)$; and (iv) the area was determined by selecting "Measure" in the Analyze menu.

In order to evaluate the DFC number, immunofluorescence confocal images of $T g(\operatorname{sox} 17: g f p) 75 \%$ epiboly embryos were merged using Image software using the following steps: (i) Images were loaded into ImageJ; (ii) "Images to stack" was selected from the "Stacks" item in the Image menu; (iii) "Stacks Focuser" was selected from the "Stacks" item in the Plugins menu. DFC number was then manually counted from images with merged GFP and nuclei. 


\section{Statistics}

Two-tailed Student's $t$-tests with unequal variance were conducted to compare number of cilia, cilia length, lumen area and DFC. To compare the klf8 mRNA rescue effect, Fisher's Exact Test was used. Statistical tests were performed with Excel software. Differences with $p<0.05$ were considered to be statistically significant.

\section{Results}

\section{klf8 morphants display abnormal heart jogging, looping} and visceral organ positions

During a previous study investigating the role of Klf8 in cerebellar development [35], we noted that klf8 morphants often exhibited a no-loop heart at $48 \mathrm{hpf}$. Because cardiac development is asymmetric, this observation suggested that Klf8 may regulate the general process of left-right patterning. Thus we performed klf8 knockdown experiments with previously validated klf8-MOs and systematically evaluated heart morphogenesis by whole-mount in situ hybridization, using a $m y l 7$ antisense RNA probe. A phenotypically normal, L-jog heart tube was readily detected in wild type and klf8-4 mm MO1- injected embryos, while embryos injected with klf8$\mathrm{MO}^{\text {atg }}$ or $\mathrm{klf}^{\mathrm{a}}-\mathrm{MO} 2^{\text {atg }}$ often showed no-jog (32\% for $\mathrm{MO}^{\text {atg }}, 33 \%$ for $\mathrm{MO}^{\text {atg }}$ ) or right-jog ( $4 \%$ for $\mathrm{MO}^{\text {atg }}, 3 \%$ for $\mathrm{MO}^{\text {atg }}$ ) heart tubes at $24 \mathrm{hpf}$ (Fig. 1a). Consequently, embryos injected with $k l f 8-\mathrm{MO}^{\text {atg }}$ or $k l f 8-\mathrm{MO} 2^{\text {atg }}$ frequently developed a no-loop heart (53\% for $\mathrm{MO}^{\text {atg }}, 40 \%$ for $\mathrm{MO}^{\text {atg }}$ ) as compared to the vast majority of wild type or klf8-4 mm MO1-injected embryos showing a D-loop heart at $48 \mathrm{hpf}$ (Fig. 1b). At $72 \mathrm{hpf}$, a similar percentage of $k l f 8-\mathrm{MO}^{\text {atg }}$ - or $k l f 8-\mathrm{MO} 2^{\text {atg }}$-injected embryos displayed a no-loop heart ( $40 \%$ for $\mathrm{MO}^{\text {atg }}, 38 \%$ for $\mathrm{MO}^{\text {atg }}$ ) as compared to wild type or klf8-4 mm MO1-injected embryos, which almost invariably had a D-loop heart (Fig. 1c). Embryos showing a delayed heart cone phenotype were also identified in the $k l f 8-\mathrm{MO}^{\text {atg }}(13 \%)$ or $k l f 8-\mathrm{MO}^{\text {atg }}(27 \%)$ groups at $24 \mathrm{hpf}$ (Fig. 1a). However, this delayed phenotype was not observed in the $48 \mathrm{hpf}$ or $72 \mathrm{hpf}$ time points. By these stages, the delayed morphants caught up developmentally and displayed either D-loop, no-loop or Lloop hearts (Fig. 1b\&c). We also examined the position of digestive organs in klf8 morphants by whole-mount in situ hybridization, using a gata6 antisense RNA probe. In
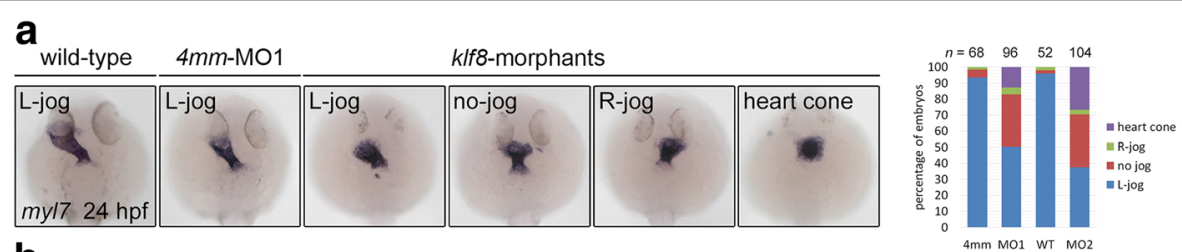

b
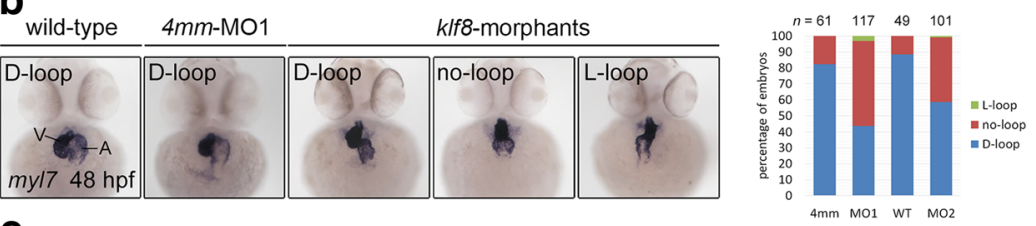

C
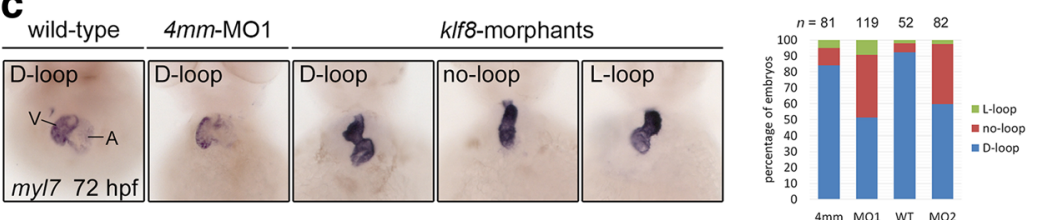

d

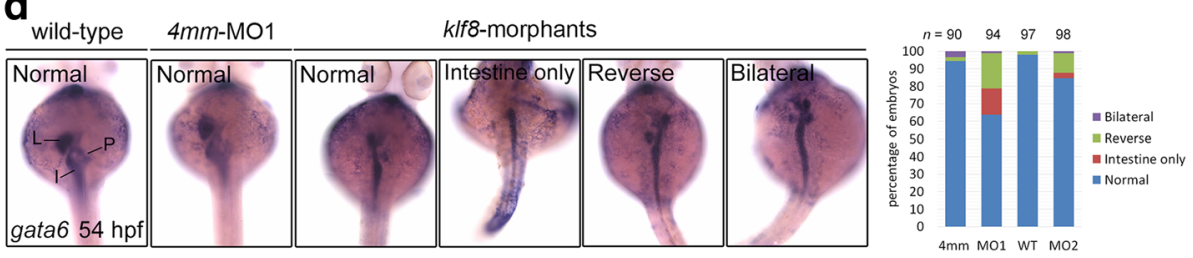

Fig. 1 Knockdown of zebrafish klf8 caused defects in heart jogging and looping, and visceral organ positions. a klf8-MO1 ${ }^{\text {atg }}$ or klf8-MO2 ${ }^{\text {atg }}$-injected embryos stained with myl7 exhibited left (L)-jog, no-jog or right (R)-jog and were compared to stained wild-type or 4 mm-MO1-injected control embryos

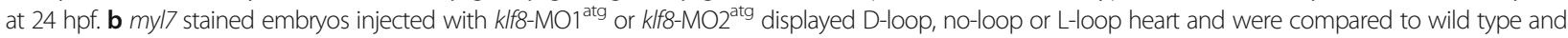
control embryos at 48 hpf. c myl7 stained embryos injected with klf8-MO1 ${ }^{\text {atg }}$ or klf8-MO2 ${ }^{\text {atg }}$ displayed D-loop, no-loop or L-loop heart and were compared to wild type and control embryos at $72 \mathrm{hpf}$. d At 54 hpf, gata6 stained wild type or embryos injected with klf8-4 mm MO1, klf8-MO1 ${ }^{\text {atg }}$ or klf8-MO2 ${ }^{\text {atg }}$ exhibited organ positions that were classified as: (Normal) normal positions of liver-left, pancreas-right and intestine with left looping, (Intestine only) only intestine without left looping, (Reverse) reversed position of liver-right, pancreas-left and no looping intestine, or (Bilateral) bilateral extension of liver and pancreas. A, atrium; I, intestine; $L$, liver; $P$, pancreas; $V$, ventricle 
$k l f 8-\mathrm{MO}^{\text {atg }}$ - or $k l f 8-\mathrm{MO}^{\text {atg }}{ }_{\text {-injected embryos, fre- }}$ quent occurrence of organ dysmorphologies were observed. Reversal of liver and pancreas position (20\% for $\mathrm{MO}^{\text {atg }}, 11 \%$ for $\mathrm{MO}^{\text {atg }}$ ), only intestine development $\left(15 \%\right.$ for $\mathrm{MO}^{\text {atg }}, 3 \%$ for $\mathrm{MO}^{\text {atg }}$ ), and bilateral liver and pancreas $\left(1 \%\right.$ for $\mathrm{MO}^{\text {atg }}, 1 \%$ for $\mathrm{MO}^{\text {atg }}$ ) were all frequently detected in $k l f 8$ morphants, but were rare events in wild type or control embryos at 54 hpf (Fig. 1d). These results confirmed that klf8 loss-of-function affects left-right patterning in zebrafish embryos.

\section{klf8 deficiency affects the level and pattern of expression for genes in the Nodal signalling pathway}

Genes encoding Nodal signalling components, including spaw, lft1, lft2, and pitx2, were asymmetrically expressed in the left side of the diencephalon, heart, or lateral plate mesoderm (LPM) during the 18-22 s stages in zebrafish embryos (Fig. 2a-m). Disruption in the expression of left-side specific Nodal signalling genes results in organ heterotaxy. We observed that spaw expression in the left LPM was either decreased (no expression in the anterior LPM and low expression in the posterior LPM, 27\%) or absent $(46 \%)$ in many $k l f 8-\mathrm{MO}^{\text {atg }}$-injected embryos (Fig. 2d-f). A similar effect (32\% decreased, 25\% absent, $8 \%$ bilateral, $5 \%$ right) was observed following klf8$\mathrm{MO}^{\text {atg }}$ injection (Fig. 2b-f). Consistent with these results, the expression of genes downstream of spaw (lft1, lft2, and pitx2) was also absent or decreased in the left diencephalon, heart, and LPM of most $k l f 8-\mathrm{MO}^{\text {atg }}$ or $k l f 8-\mathrm{MO} 2^{\text {atg }}$-injected embryos (Fig. 2h-o). Additionally, we injected three splicing $\mathrm{MOs}\left(\mathrm{MO}^{\mathrm{DO}}, \mathrm{MO}^{\mathrm{AC}}\right.$, and $\mathrm{MO}^{\text {Do }}$ ) to block splicing of klf8 mRNA (Additional file 1: Figure S1, A). RT-PCR indicated that splicing of klf8 mRNA was effectively disrupted in $\mathrm{MO}^{\mathrm{sp}-\mathrm{MOs}}$-injected embryos at 24 hpf (Additional file 1: Figure S1, B). Expression of spaw in the left LPM was either decreased $(8 \%)$ or eliminated $(63 \%)$ in most $18 \mathrm{~s}$ stage embryos injected with $\mathrm{MO}^{\mathrm{sp}-\mathrm{MOs}}$ (Additional file 1: Figure S1, C).

Klf8 was previously shown to repress $p 53$ expression and induce met expression to modulate the development of Purkinje cells and proliferation of granule cells [35]. To confirm that defective left-right patterning did not arise from induction of $p 53$ due to klf8 deficiency, we analysed heart looping and gene expression of Nodal signalling components in embryos that were co-injected with $p 53-\mathrm{MO}^{\mathrm{sp}}$, alongside $k l f 8-\mathrm{MO}^{\text {atg }}$ or $k l f 8-\mathrm{MO}^{\text {atg }}$.

Of the embryos co-injected with $p 53-\mathrm{MO}^{\mathrm{sp}}$, together with $k l f 8-\mathrm{MO}^{\text {atg }}$ or $k l f 8-\mathrm{MO}^{\text {atg }}, 35-39 \%$ exhibited a no-loop heart at $72 \mathrm{hpf}$, similar to embryos injected with klf8 MOs alone (Additional file 2: Figure S2, A). Likewise, expression levels of spaw, lft1, lft2, and pitx2 were reduced or eliminated in the left LPM, diencephalon, and heart of high percentages of co-injected embryos

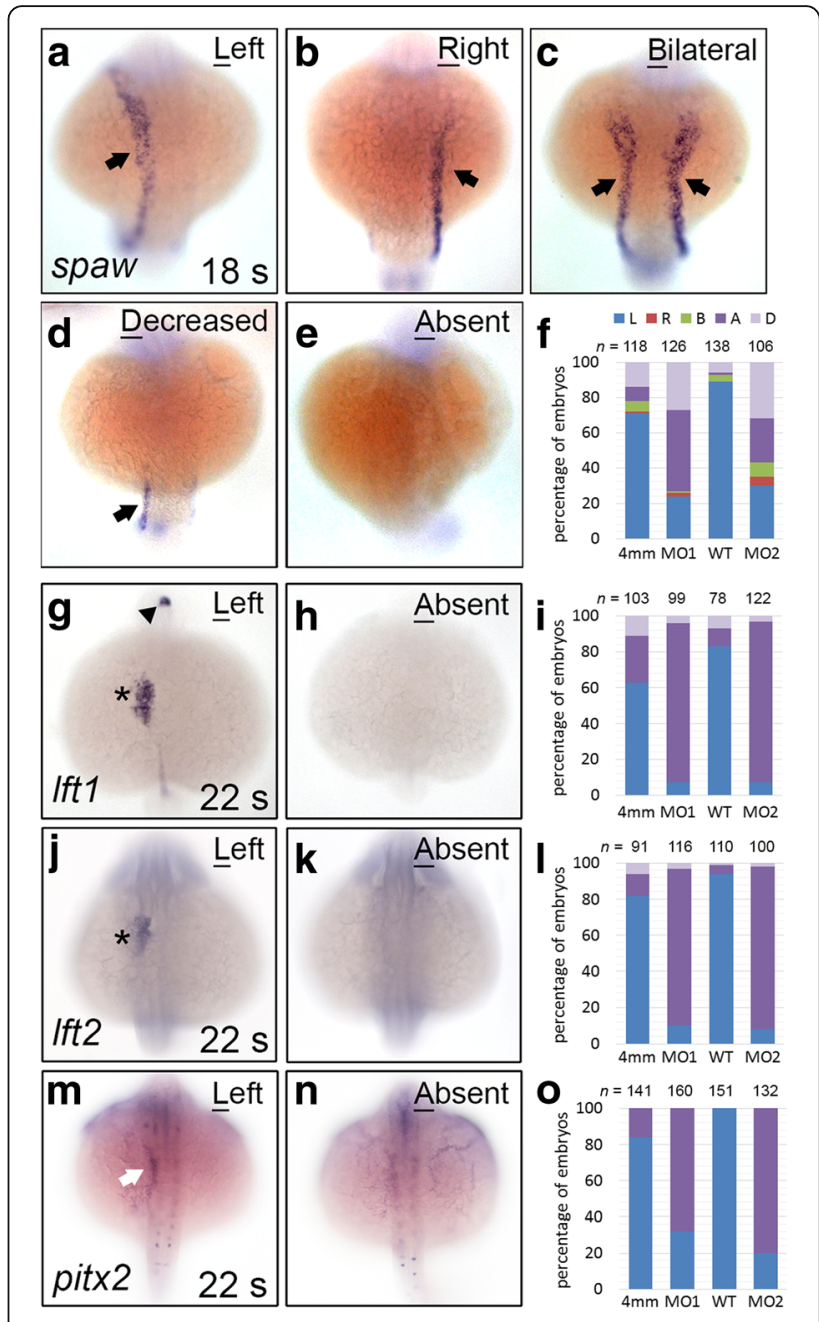

Fig. 2 Genes encoding Nodal signalling components exhibited decreased or abolished expression in klf8 morphants. The majority of klf8-MO1 $1^{\text {atg }}$ or klf8-MO2 ${ }^{\text {atg }}$-injected embryos showed decreased or abolished spaw (arrow) expression in the left lateral plate mesoderm (LPM) at the $18 \mathrm{~s}$ stage (a-f). The majority of klf8 morphants failed to express Ift1 in the left diencephalon (arrowhead) or heart (asterisk) $(\mathbf{g}-\mathbf{i})$, Ift2 in the left heart (asterisk) (j-l), and pitx2 in the left LPM (white arrow) $(\mathbf{m}-\mathbf{0})$ at the $22 \mathrm{~s}$ stage. Dorsal views of embryos are shown. A, absent; B, bilateral; D, decreased; L, left; R, right

during the 18-22 s stages (Additional file 2: Figure S2, B-E). We also found that the expression levels of gata5 and oep (which are known to be expressed in the LPM at the $22 \mathrm{~s}$ stage) were unaffected by klf 8 knockdown (Additional file 2: Figure S2, F-M). Together these data clearly indicate that increased p53 expression and apoptosis are not responsible for the decreased expression of genes involved in Nodal signalling.

To further confirm that the decrease in spaw expression is a consequence of klf8 loss-of-function, we performed rescue experiments by co-injecting embryos with $k l f 8-\mathrm{MO} 1^{\text {atg }}$ and $k l f 8$ mRNA. Approximately $69 \%$ of $k l f 8-\mathrm{MO}^{\text {atg }}$-injected embryos exhibited eliminated or 
decreased spaw expression in the left LPM at the $18 \mathrm{~s}$ stage (Fig. 3a, e). However, this proportion showed a statistically significant reduction to $44 \%$ for embryos coinjected with $k l f 8-\mathrm{MO}^{\text {atg }}$ and $k l f 8$ mRNA (Fig. 3b-e). Taken together, these results demonstrate that klf8 lossof-function causes downregulation of Nodal signalling component genes.

\section{Overexpression of klf8 mRNA causes bilateral expression of genes involved in Nodal signalling}

Since klf8 knockdown reduced expression of genes involved in Nodal signalling, we hypothesized that $k l f 8$ overexpression may have the opposite effect. While the majority of embryos injected with $100 \mathrm{pg}$ of LacZ mRNA expressed spaw exclusively in the left LPM at the $18 \mathrm{~s}$ stage, $24 \%$ of embryos injected with $50 \mathrm{pg}$ and $51 \%$ of embryos injected with $100 \mathrm{pg}$ of klf8 mRNA expressed spaw bilaterally in the LPM (Fig. 4c, e). Thus, a dose-dependent effect of klf8 expression was revealed. Moreover, embryos overexpressing klf8 also frequently exhibited bilateral expression patterns of lft 1 , lft2, and pitx 2 in the diencephalon, heart, and LPM at 19-22 s stages (Fig. 4h-t). On the other hand, $n t l$ expression in the notochord was not altered in $22 \mathrm{~s}$ stage embryos overexpressing klf8 as compared to LacZ-overexpressing embryos (Fig. $4 \mathrm{u}-\mathrm{w}$ ). In order to investigate whether the zinc finger DNA binding domain of Klf8 is involved in regulating the expression pattern of spaw or its downstream genes, we injected mRNA for klf8 lacking the zinc finger DNA binding domain $(k l f 8 \Delta z f)$. We found that injection of $100 \mathrm{pg} k l f 8 \Delta z f$ only induced a low percentage of embryos to exhibit bilateral expression of spaw (6.3\%), lft1 (3.6\%), lft2 (1.6\%) or pitx2 (11.9\%), compared to higher rates of bilateral spaw (48.3\%), lft1 (31.6\%), lft2 (37.5\%) or pitx2 (44.3\%) expression in klf8 injected embryos at $18 \mathrm{~s}$ or $19-22 \mathrm{~s}$ stages (Additional file 3: Figure S3). These results demonstrate

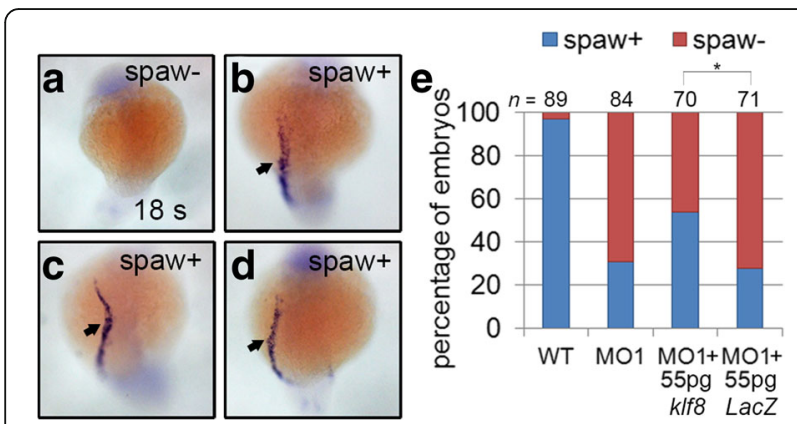

Fig. 3 Reduced spaw expression in klf8 morphants was partially rescued by co-injection of klf8 mRNA. Representative embryos showing spaw expression in the left LPM (spaw+) or absent spaw expression (spaw-) are shown (a-d). Percentages of embryos with asymmetric spaw expression or no spaw expression are shown with indicated treatments (e). Statistical significance was determined by Fisher's Exact Test. ${ }^{*} p<0.05$ that overexpression of klf8 does not affect the midline structure, but induces ectopic expression of spaw and its downstream genes when the Klf8 zinc finger DNA binding domain is intact.

\section{klf8 deficiency affects morphogenesis of Kupffer's vesicle and asymmetric charon expression}

Since asymmetric flow, generated by rotation of cilia within $\mathrm{KV}$, is essential to initiate left-right asymmetric patterning, and $\mathrm{KV}$ is derived from dorsal forerunner cells (DFCs), we then investigated whether klf8 knockdown affected cilia or DFC number during KV morphogenesis. Individual $k l f 8-\mathrm{MOs}$ or control $\mathrm{MO}$ were microinjected into one- or two-cell $T g(\operatorname{sox} 17: g f p) \mathrm{zy}-$ gotes, and immunofluorescence was conducted using anti-GFP antibody. We found that the number of DFCs at the dorsal margin was significantly reduced in klf8$\mathrm{MO}^{\text {atg }}$ - (average of $26.6 \mathrm{DFCs}$ ) or $k l f 8-\mathrm{MO}^{2 \text { atg }}$ - (average of 27.6 DFCs) injected $\mathrm{Tg}$ (sox17:gfp) embryos as compared to wild type (average of $36.2 \mathrm{DFCs}$ ) or klf8-4 mm MO1- (average of 35.7 DFCs) injected embryos at $75 \%$ epiboly (Fig. 5a-d, o). Cilia were then detected by immunofluorescence staining of 10s stage embryos using an anti-acetylated tubulin antibody. KV lumen size was smaller, but not significantly so, in $\operatorname{Tg}($ sox $17: g f p)$ embryos injected with $k l f 8-\mathrm{MO}^{\text {atg }}, k l f 8-\mathrm{MO}_{2}^{\text {atg }}$, or $\mathrm{MO}^{\mathrm{sp}-\mathrm{MOs}}$ as compared to wild type or klf8-4 mm MO1-injected control embryos at 10s stage (Fig. 5e-n, p). Significantly reduced number and length of KV cilia were detected in embryos injected with different $k l f 8-\mathrm{MOs}$ as compared to wild type and control embryos (Fig. 5q, r). Since asymmetric charon expression on the right side of the $\mathrm{KV}$ was influenced by strength and direction of KV flow [40], we also examined whether klf8 knockdown affected asymmetric charon expression around KV. The majority $\left(61 \%\right.$ for $\mathrm{MO}^{\text {atg }}, 57 \%$ for $\mathrm{MO}^{\text {atg }}$ ) of embryos injected with different klf8-MOs revealed symmetric charon expression with reduced expression area around KV as compared to wild type and control embryos at the 10s stage (Additional file 4: Figure S4). These results indicate that KV morphogenesis, cilia length, cilia number and asymmetric charon expression were affected in klfs knockdown embryos.

\section{Generation of klf8 mutant by a CRISPR-Cas9 system}

In order to confirm our morphant results, we generated klf8 mutants using a CRISPR-Cas9 system. Although we designed three $k l f 8$ sgRNAs targeting to exon 2 or exon 3 , only klf8 sgRNA1, which targets to exon 2, was successful in producing mutants. Administration of sgRNA1 induced efficient deletion or insertion of bases in exon 2 and resulted in two klf8 mutant alleles ( $k l f \delta^{d 25}$ and $k l f 8^{i 17}$ ) (Fig. 6a). The klf8 $8^{d 25}$ mutant had a 25 bp deletion, which produced a 35 amino acid-long misframed Klf8 


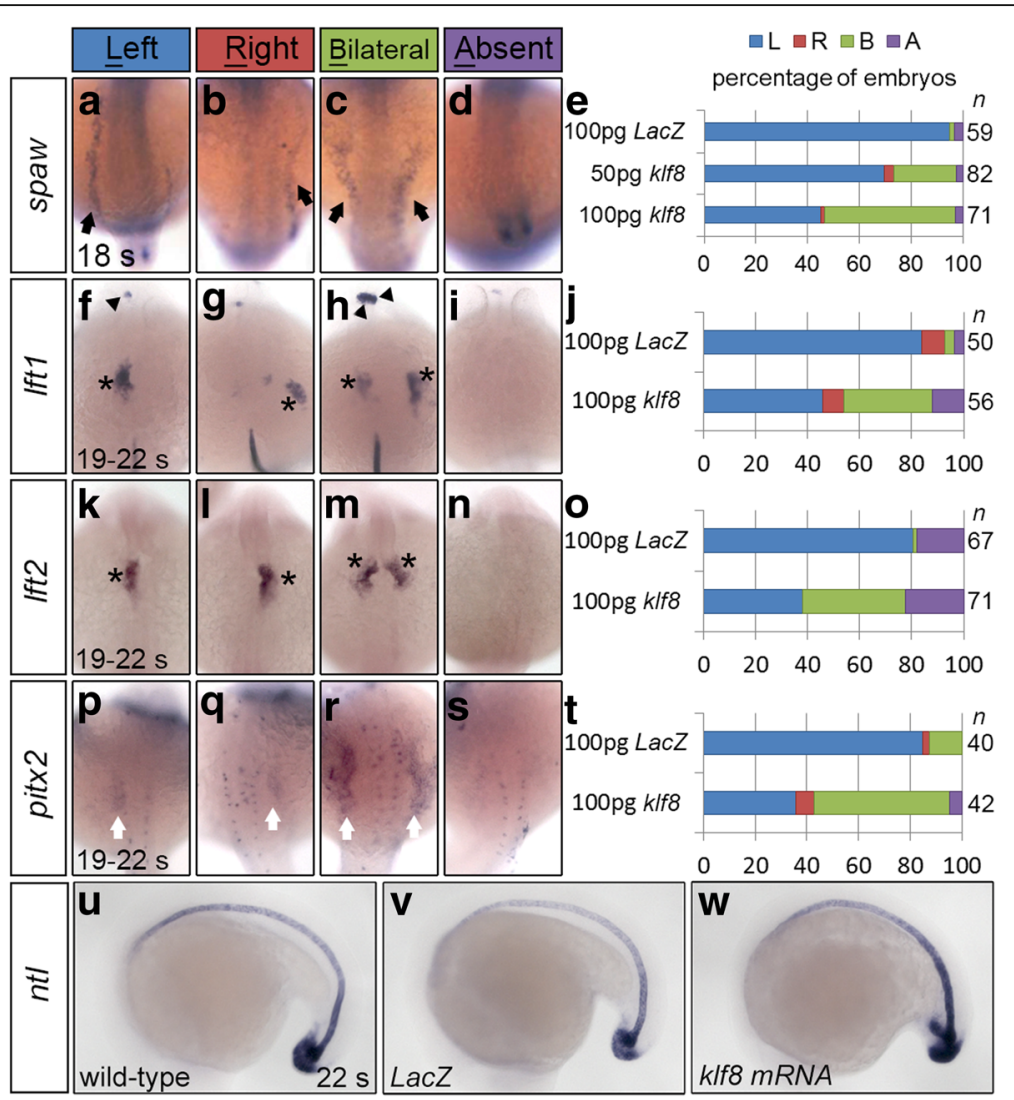

Fig. 4 Overexpression of klf8 mRNA caused bilateral expression of Nodal signalling component genes. Injection of klf8 mRNA induced bilateral expression of spaw in the LPM (arrow) at the $18 \mathrm{~s}$ stage, in a dose-dependent manner (a-e). Embryos injected with klf8, but not LacZ, exhibited bilateral expression of Ift 1 in the diencephalon (arrowhead) and heart (asterisk; $\mathbf{f - j}$ ), Ift2 in the heart (asterisk; $\mathbf{k}-\mathbf{o})$, and pitx2 in the LPM (white arrow) (p-t) at the 19-22 s stage. Expression of $n t /$ in the notochord is similar in wild type and embryos injected with LacZ or klf8 mRNA (u-w). A, absent; $B$, bilateral; $L$, left; $R$, right

protein, while the $k l f 8^{i 17}$ mutant had a 17 bp insertion that generated a 49 amino acid-long misframed Klf8 protein (Fig. 6b).

Of note, in 24 hpf homozygous F3 embryos of $k l f 8^{d 25}$ and $k l f 8^{i 17}$ mutants, we did not observe smaller eyes and abnormal cerebellar morphology that were detected in klf8 morphant embryos [35]. Next, we investigated whether spaw expression was affected in klf8 mutant embryos. Bilateral spaw expression was more frequently observed in embryos from the intercross of respective $k l f 8^{d 25}$ (27 out of $69,39.1 \%$ ) or $k l f f^{i 17}$ (9 out of 35, 25.7\%) F2 heterozygous mutants as compared to wild type embryos (7\%) at $18 \mathrm{~s}$ stage (Fig. 6c-g). In order to evaluate the genotype of F3 embryos from $k_{l f} 8^{i 17}$ and $k l f 8^{d 25}$ mutants with bilateral spaw expression, we sequenced seven $k_{l f f} 8^{i 17}$ and $18 \mathrm{klfg}^{d 25}$ embryos with the phenotype. From sequencing data, we obtained two (28.6\%) wild type, three (42.9\%) heterozygotes and two (28.5\%) homozygotes

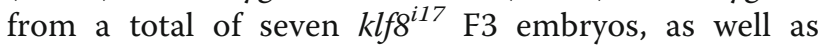
three $(16.7 \%)$ wild type, seven $(38.9 \%)$ heterozygotes and eight (44.4\%) homozygotes from a total of 18 $k l f 8^{d 25}$ embryos. We then deduced that $7.3 \%$ of $k l f 8^{i 17}$ or $17.4 \%$ of $k l f 8^{d 25} \mathrm{~F} 3$ embryos were homozygous mutant embryos that also had bilateral spaw expression. This observation was based on the following calculation $[0.285$ (\% of sequenced embryos that were homozygous) $\times 9$ (total number with bilateral spaw expression) / 35 (total number of embryos) $=7.3 \%$ for $k l f 8^{i 17} ; 0.444 \times 27 / 69=17.4 \%$ for $\left.k l f 8^{d 25}\right]$. The other F3 embryos that exhibited bilateral spaw expression were also deduced to be either heterozygous mutant embryos (11\% in klf $8^{i 17}, 15.2 \%$ in $\left.k l f 8^{d 25}\right)$ or sibling wild type $\left(7.4 \%\right.$ in $k l f 8^{i 17}, 6.5 \%$ in $\left.k l f 8^{d 25}\right)$ based on similar calculations.

Because human KLF8, KLF3 and KLF12 form a subgroup in phylogenetic tree analysis due to the presence of CtBP-binding sites [41], we wondered whether expression of zebrafish klf3, klf12a, or klf $12 b$ may be upregulated to compensate for klf8 deficiency. We discovered substantial upregulation of $k l f 12 b$ in $k_{l f} 8^{d 25} \quad \mathrm{~F} 5$ homozygous mutant embryos and 

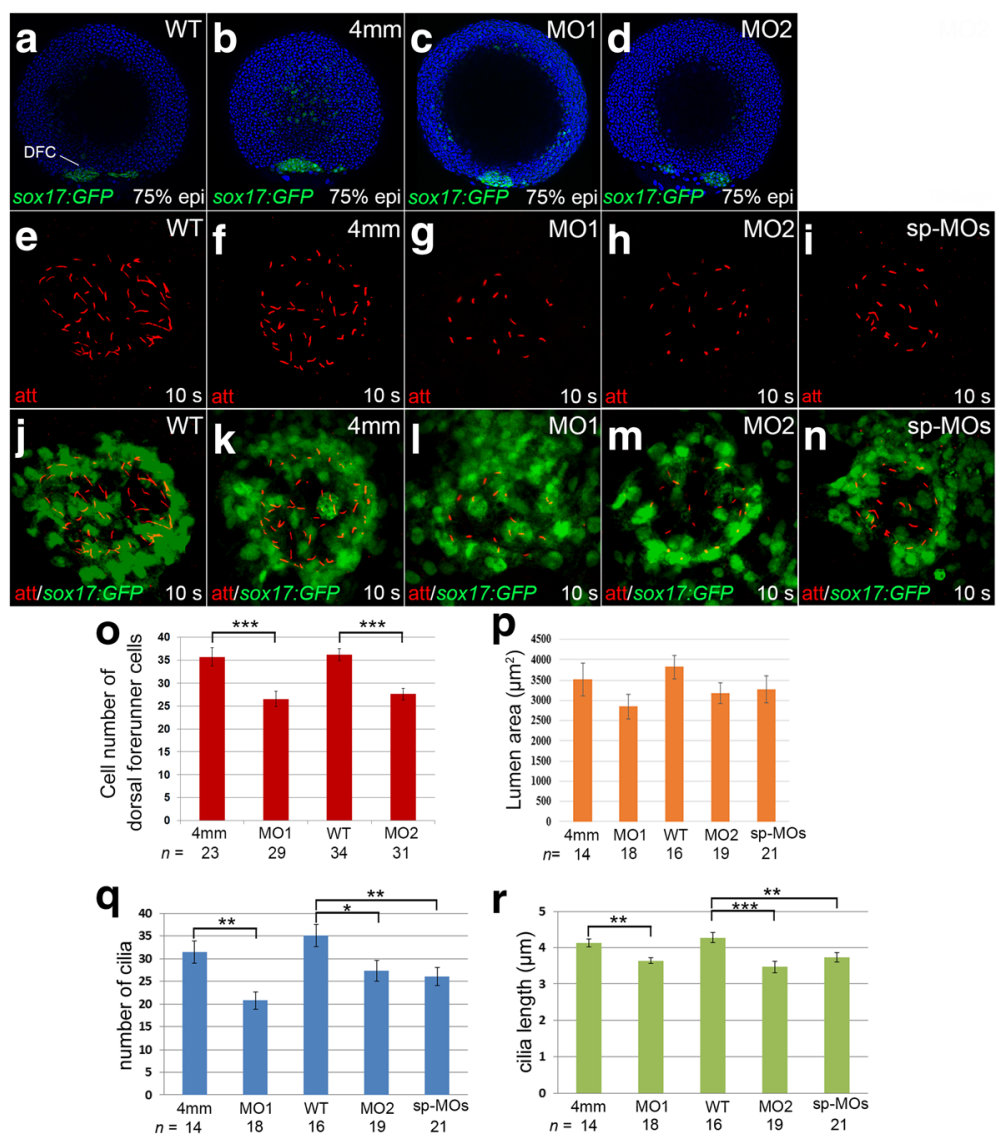

Fig. 5 Knockdown of klf8 affected dorsal forerunner cell number, KV cilia number and length. Cell number of dorsal forerunner cells (DFCs) was affected in klf8-MO1 ${ }^{\text {atg }}$ - or klf8-MO2 ${ }^{\text {atg }}$-injected embryos as compared to klf8-4 mm MO1-injected Tg(sox17:gfp) control embryos (4 mm) at 75\% epiboly (a-d). Images of KV cilia stained with acetylated tubulin antibody in klf8 morphants ( $\mathbf{g}-\mathbf{i})$ and control embryos (e, $\mathbf{f})$ at 10 s stage (e-i). Images of acetylated tubulin stained KV cilia and GFP stained DFCs in Tg(sox17:gfp) embryos injected with different klf8 MOs (I-n), klf8-4 mm MO1 (k) or wild type (j) embryos at 10s stage (j-n). DFC number (o), KV cilia number (q), length (r) but not lumen area (p) were affected in klf8 morphants as compared to control embryos. Statistical significance was determined by Student's t-test. ${ }^{*} p<0.05,{ }^{* *} p<0.01,{ }^{* * *} p<0.001$. Error bars indicate standard deviation

downregulation of $k l f 12 a$ in $k l f 8^{i 17}$ F5 homozygous mutant embryos at 10-12 s stages, while no alteration of klf3 expression was observed in either $k_{l f f} 8^{d 25}$ or $k l f 8^{i 17}$ mutant embryos (Fig. 6h-m). Subsequently, we knocked down $k l f 12 b$ in $k l f 8^{d 25}$ F6 homozygous mutant embryos and evaluated the spaw expression pattern at $18 \mathrm{~s}$ stage (Fig. 6n). In klf ${ }^{d 25}$ F6 homozygous mutant embryos, bilateral (50.3\%) and decreased (6.3\%) spaw expression patterns were detected. In homozygous mutant embryos injected with 5 or 10 ng klf $12 b \mathrm{MO}$, we found a dose-dependent reduction that did not reach significance in the percentage $(42.1 \%$ for $5 \mathrm{ng}, 29.3 \%$ for $10 \mathrm{ng}$ ) of embryos with bilateral spaw expression, which was accompanied by the increased occurrence of right ( $1.6 \%$ for $5 \mathrm{ng}, 1.7 \%$ for $10 \mathrm{ng})$, decreased ( $15.9 \%$ for $5 \mathrm{ng}, 16.4 \%$ for $10 \mathrm{ng}$ ) or absent (4.0\% for $5 \mathrm{ng}, 3.4 \%$ for $10 \mathrm{ng}$ ) spaw expression patterns.

These results indicate that although klf8 mutant embryos display different spaw expression patterns than morphant embryos, this effect may be partly attributed to a compensatory induction of $k l f 12 b$ expression.

\section{Discussion}

Establishing asymmetric spaw expression in the LPM is essential for left-right patterning in zebrafish embryos. Expression of spaw is first apparent in bilateral cells flanking KV between the 4-6 s stages, while asymmetric spaw expression emerges in the posterior LPM during the 10-12 s stages, and extends to the anterior LPM by the $18 \mathrm{~s}$ stage [22]. Knockdown of spaw abolishes spaw expression in the LPM but not in peri-KV domains [21], suggesting that autoregulation of spaw occurs only in the left LPM. We used morpholino antisense oligomers to knockdown klf8, and found that spaw expression in the left LPM was reduced or eliminated in the majority of 18-22 s stage morphants (Fig. 2, Additional file 2: Figure S2). Spaw activates expression of itself, as well as lft1, lft2, and pitx2 in the left LPM during the 
a
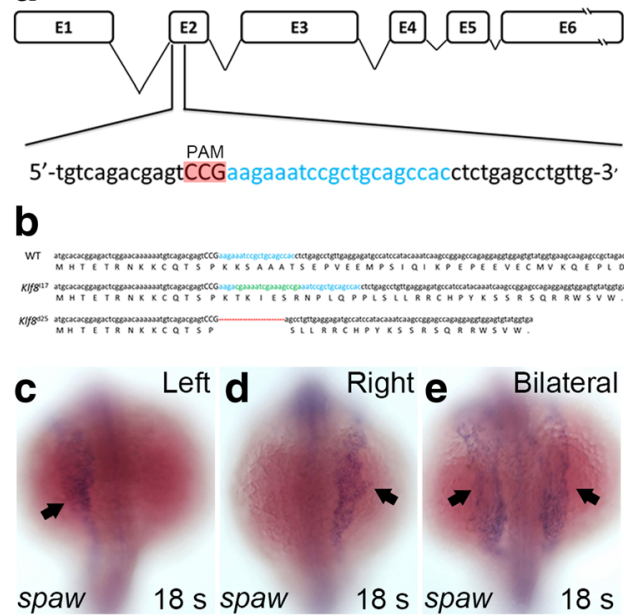
f Decreased $\mathbf{9}_{100}^{n=103}$

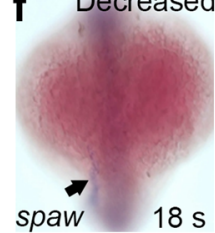

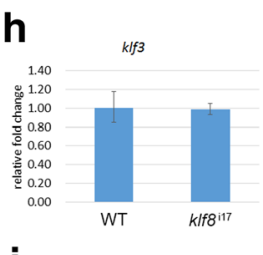

i

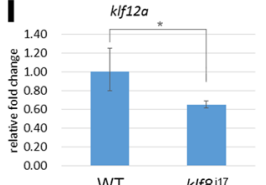

WT

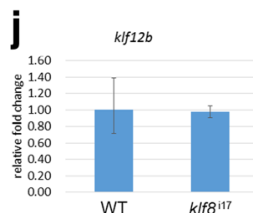

$\mathbf{n}_{100}=159 \quad$ KIf8

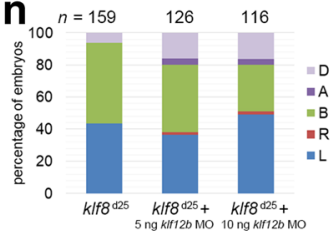

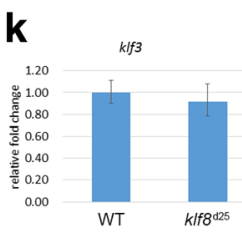

| $k l f 12 a$

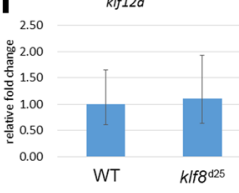

m $k l f 12 b$

2.00
$\stackrel{2}{0} 1.50$

递 1.50

응 1.00

屁 0.50

WT

$$
\begin{aligned}
& =D \\
& =A \\
& =B \\
& =R \\
& =L
\end{aligned}
$$

Fig. 6 Generation of klf8 mutants by CRISPR-Cas9 gene editing and the effect on spaw expression. a klf8 genomic structure with klf8 sgRNA (blue lettering) targeted to exon 2. Protospacer adjacent motif (PAM) sequence is shown in red. $\mathbf{b}$ Nucleotide and predicted amino acid sequences of klf8 in wild type, $k_{1 f 8^{117}}$ and klf8d25 mutants are shown. Deleted nucleotides are shown by a red dashed line, while inserted nucleotides are shown in green lettering. Representative images of embryos with different spaw expression patterns in the LPM at $18 \mathrm{~s}$ stage (arrow; c-f). $\mathbf{g}$ Percentage of embryos displayed left (L), right (R), decreased (D) or bilateral (B) expression of spaw in the LPM from intercross of respective klf8 ${ }^{d 25}$ or klf8 ${ }^{i 7}$ F2 $^{2}$ heterozygous mutants. Deduced percentage of wild type (+/+), heterozygote (+/-) or homozygote (-/-) genotype of embryos from intercross of respective $k 1 f 8^{d 25}$ or $k 1 f 8^{i 17} \mathrm{~F} 2$ heterozygous mutants exhibited bilateral spaw expression pattern. Expression levels of $k l f 3$ (h, k), klf12a (i, I) or klf12b $(\mathbf{j}, \mathbf{m})$ were compared between wild type and respective klf8 ${ }^{d 25}$ and klf8 ${ }^{i 7}$ F5 homozygous mutant embryos at $10-12$ s stages (h-m). Knockdown of $k 1 f 12 b$ reduced the percentage of embryos with bilateral spaw expression in the LPM of klf8 ${ }^{d 25} \mathrm{~F} 6$ homozygous mutant embryos, but the reduction did not reach significance $\left(p=0.45\right.$ for the comparison between $k 1 f 8^{d 25}$ and $k 1 f 8^{d 25}+5 \mathrm{ng} k 1 f 812 b \mathrm{MO}, p=0.05$ for the comparison between $k l f 8^{d 25}$ and $\left.k l f 8^{d 25}+10 \mathrm{ng} k l f 812 b \mathrm{MO}\right)(\mathbf{n})$. Statistical significance was determined by Student's $t$-test. * $p<0.05$. Error bars indicate standard deviation

segmentation stage [22]. As such, the observed reduction or elimination of $l f t 1, l f t 2$, and pitx 2 in the left diencephalon, heart or LPM at 18-22 s stages in the majority of klf8 morphants was not unexpected (Fig. 2, Additional file 2: Figure S2). Overall, defects in the expression of spaw and its downstream genes, and the subsequent defects in internal organ patterning observed in klf8 morphants are consistent with those detected in spaw morphants [22]. Although spaw/sfw mutant embryos more frequently displayed a D-loop heart $(68 \%)$, compared to klf8 morphants, the difference may be attributed to heart specific actomyosin activity. Furthermore, in $s f w$ mutant embryos, expression of laterality genes including $l f t 1$, lft2, and pitx2 were lost, and spaw expression did not propagate toward the anterior of the left LPM [42]. In our study, we saw that overexpression of $k l f 8$ but not $k l f 8 \triangle z f$ resulted in bilateral expression of spaw, lft1, lft2, and pitx 2 at $18 \mathrm{~s}$ or 19-22 s stages (Fig. 4, Additional file 3: Figure S3), demonstrating a requirement for the Klf8 zinc finger DNA binding domain. Expression of $n t l$ in the notochord was found to be unaltered in klf8-overexpressing embryos, suggesting that the midline structure is in intact. Overall, our results demonstrate that Klf8 may regulate asymmetric spaw expression in the left LPM, which in turn affects left-side specific expression of lft1, lft2, and pitx2 in zebrafish embryos.

Proper morphogenesis of KV is also important for initial asymmetric spaw expression in the posterior LPM. The development of KV involves the formation of DFCs from surface epithelial cells, ingression at the dorsal germ ring margin, DFC migration, formation of rosettelike epithelial structures, coalescence of epithelial rosettes, and differentiation of ciliated KV with interior lumen $[15,43]$. Both Tbx16 and Ntl were shown to regulate a mesenchymal to epithelial transition that participates in the formation of rosette-like epithelia [44]. Wnt11- and Prickle1a-mediated planar cell polarity signalling, as well as Cnpy1-mediated FGF signalling, were shown to regulate cell adhesion between adjacent dorsal 
forerunner cells to maintain cluster formation [45, 46]. Defects in these signalling events resulted in small KV lumen, with shortened and decreased number of KV cilia. In klf8 morphants, a similar smaller KV lumen, with decreased number and length of KV cilia was frequently observed at the 10 s stage. These abnormal structures may result from lower number of DFCs that was observed in $75 \%$ epiboly morphants (Fig. 5). Whether Klf8 may participate in Wnt11- and Prickle1a-mediated planar cell polarity signalling, or Cnpy1-mediated FGF signalling to modulate DFC cluster formation, remains to be determined.

Zebrafish KV architecture is asymmetric along the anterior-posterior axis, with more ciliated cells in the anterior region. Furthermore, the positioning of the basal body of motile cilia at the posterior end of the epithelial cells may result in cilia tilting $[17,47]$. These motile cilia then use a vortical motion to generate swirling fluid flow consisting of a relatively stronger leftward flow across the anterior pole of $\mathrm{KV}$ and a weaker rightward flow at the posterior end $[16,18]$. Based on experimental tracking of native particles within the KV of wild type, did $^{-/-}$mutant and dnah7 morphants, and simulated flow by mathematically modelling, it was determined that a threshold of 30 cilia, with dorsal anterior clustering, is essential to generate proper swirling flow in the anti-clockwise direction [40]. In control embryos, with strong left-sided flow across the anterior pole of $\mathrm{KV}$, asymmetric expression is established for charon in the right side of the KV, and spaw in the left LPM. In embryos with non-directional flow, symmetric charon expression and a lack of spaw expression may be found. Embryos without motile cilia, and therefore no KV flow, may exhibit symmetric and slightly weaker charon expression and bilateral spaw expression in the posterior LPM [40]. In klf8 morphant embryos, injected with different klf8 MOs, a significantly reduced number of cilia $(<30)$, with random distribution was detected (Fig. 5). KV with such a cellular architecture may exhibit a weak and homogenous fluid flow, resulting in the symmetric charon expression around $\mathrm{KV}$ that was detected in the majority of klf8 morphants, and leading to drastically reduced spaw expression at late somite stage (Additional file 4: Figure S4, Fig. 2). Overall, our results clearly indicate that Klf8 is required for normal KV morphogenesis, which is known to be critical for initiating asymmetric spaw expression in the left LPM.

In mouse and chick, BMP signalling plays either a positive or negative role in regulating asymmetric Nodal expression. Moreover, the presence of BMP antagonists, such as Noggin, Chordin, or Caronte, can relieve BMPinhibition to promote asymmetric Nodal expression in the left LPM [48-51]. In zebrafish embryos, heat-activated $B M P 2 b$ expression inhibits spaw expression, while heat-activated noggin3 induced bilateral spaw expression, indicating that BMP signalling is required to repress spaw expression in the right LPM of early segmentation stage embryos, [25]. In addition, expression of Lft1 in the midline, and Lft2 in the left cardiac field, serve to generate a posterior or anterior barrier to restrict Spaw activity to the left LPM during segmentation stages in zebrafish embryos [27]. Dvr1, a zebrafish Vg1 ortholog, was also shown to facilitate the transfer of spaw expression from the peri-KV region to the left LPM. Thus, reduced or absent expression of spaw and downstream lft 1 and lft 2 in the LPM, diencephalon, notochord, or heart were detected in $d v r 1$ morphants [52]. In order to investigate whether Klf8 may regulate asymmetric spaw expression via modulation of expressions of $b m p 2 b$ or $d v r 1$, we then compared expression of these two genes between klf8 morphants and control embryos (Additional file 5: Figure S5). Similar $b m p 2 b$ expression level around tailbud region was identified in $3 \mathrm{~s}$ stage wild type and embryos injected with klf8$\mathrm{MO}^{\text {atg }}, k l f 8-\mathrm{MO}^{\text {atg }}$ or klf8- $4 \mathrm{~mm} \mathrm{MO1}$. Likewise, no alteration of $d v r 1$ expression around the tailbud region was detected in wild type and embryos injected with $k l f 8-\mathrm{MO}^{\text {atg }}, k l f 8-\mathrm{MO} 2^{\text {atg }}$ or $k l f 8-4 \mathrm{~mm}$ MO1 at bud stage. Thus, Klf8 does not act via $\mathrm{BMP} 2 \mathrm{~b}$ or Dvr1 signalling pathway to regulate asymmetric spaw expression, and the underlying mechanism remains to be determined.

In addition to our studies with $k l f 8$ morphants, we generated $k l f 8$ mutants by a CRISPR-Cas9 system (Fig. 6). Intriguingly, obvious phenotypic differences were found between morphants and mutants. In 24 hpf homozygous F3 embryos of $k l f 8^{d 25}$ and $k l f 8^{i 17}$ mutants, we did not observe smaller eyes and abnormal cerebellar morphology that were detected in klf8 morphant embryos [35]. In addition, bilateral spaw expression was detected in the LPM of $k l f 8^{d 25}$ and $k l f 8^{i 17}$ mutants at the $18 \mathrm{~s}$ stage (Fig. 6). Discrepant phenotypes between mutants created by TALENs or CRISPR-Cas genome editing systems and antisense morpholino mediated-morphants have been frequently encountered. Previously, differences have been attributed to off-target effects of morpholinos [53], or compensatory effects, which have been described in vasculature development [54], reproduction [55], or neurogenesis [56]. With regard to the two klf8 mutant alleles from our study, more $k l f 8^{d 25}$ mutant embryos showed bilateral spaw expression in the LPM, compared to $k l f 8^{i 17}$ mutants (Fig. 6g, n). This difference in outcome may correlate with aberrant upregulation of $k l f 12 b$ in $k l f 8^{d 25}$ that was further confirmed by $k l f 12 b$ knockdown, but not in $k l f 8^{i 17}$, mutants (Fig. 6n). Similar compensatory inconsistency was found in stmn 4 mutants, which had a low $(<10 \%)$ portion of embryos showing similar phenotype to stmn 4 morphants. Interestingly, the authors found that stmn1b was upregulated to 
compensate in $s t m n 4 \Delta 5$ but not stmn $4 \Delta 4$ mutants [56]. In our study, we observed that in response to klf8 deficiency, $k l f 12 b$, a member of a subgroup of KLF family with a CtBP interaction site, was induced to compensate for the loss of $k l f 8$. However aberrant upregulation of $k l f 12 b$ further resulted in bilateral spaw expression. On the contrary, downregulation of $k l f 12 a$ was detected in $k l f 8^{i 17}$ mutants (Fig. 6). In these mutants, bilateral spaw expression was observed to a lesser degree, suggesting that $k l f 12 a$ may have undergone functional divergence with $k l f 12 b$, and as such, $k l f 12 a$ may play a role in restricting spaw expression to the left side of embryos. Overall, klf8 mutant embryos showed bilateral spaw expression, which was quite different from klf8 morphants that exhibited reduced or eliminated spaw expression in the LPM. This dissimilar phenotype may have been partly related to the compensatory induction of $k l f 12 b$ expression in the mutant embryos.

\section{Conclusions}

In this report, we have demonstrated a novel role for zebrafish Klf8 in left-right asymmetric patterning. During gastrulation, Klf8 may regulate DFC cell number to control proper KV morphogenesis, which is essential to initiate asymmetric spaw expression in the left LPM. During somitogenesis, Klf8 may further modulate asymmetric spaw expression in the left LPM to ensue asymmetric organ positioning.

\section{Additional files}

Additional file 1: Figure S1. Knockdown of klf8 expression by splicing morpholino oligomers resulted in embryos with reduced or absent spaw expression in the left LPM. A Klf8 genomic structure showing position of translational morpholino oligomers (klf8-MO1 ${ }^{\text {atg }}$, klf8-MO2 $^{\text {atg }}$ ) and splicing morpholino oligomers $\left(k_{f} 8^{\mathrm{DO} 2}, \mathrm{kff} 8^{\mathrm{AC}}, \mathrm{klf8} \mathrm{DO}^{\mathrm{O}}\right)$. Arrows indicate the positions of forward and reverse primers. B RT-PCR showing the efficacy of klf8 splicing morpholino oligomers. C The majority of embryos injected with kffs splicing MOs had decreased or absent spaw expression in the left LPM. (TIFF $153 \mathrm{~kb}$ )

Additional file 2: Figure S2. Heart looping and downregulated expression of spaw and its downstream genes were not caused by induction of p53 expression in Klf8 morphants. Embryos co-injected with p53-MO ${ }^{\text {sp }}$ and Klf8-MO1 $^{\text {atg }}$ or klf8-MO2 ${ }^{\text {atg }}$ displayed no-loop or L-loop heart defects at $72 \mathrm{hpf}$ (A). The majority of embryos co-injected with $\mathrm{p} 53-\mathrm{MO}^{\mathrm{Sp}}$ and klf8-MO1 ${ }^{\text {atg }}$ or klf8-MO2 $2^{\text {atg }}$ exhibited decreased or absent expression of spaw (B), Ift1 (C), Ift2 (D), or pitx2 (E) in the left LPM, diencephalon or heart at the 18-22 s stages. Expression levels of gata5 and oep which are known to be expressed in the LPM at the $22 \mathrm{~s}$ stage were unaffected by klf8 knockdown (F-M). (TIFF $797 \mathrm{~kb}$ )

Additional file 3: Figure S3. Overexpression of klf8 but not klf8 $\Delta_{Z} f$ mRNA induced bilateral expression of Nodal signalling component genes. Percentages of embryos injected with either $100 \mathrm{pg}$ of $\mathrm{klf8}$ or klf8 $\triangle \mathrm{zf}$ mRNA that exhibit left $(\mathrm{L})$, right $(\mathrm{R})$, decreased (D) or bilateral (B) expression of spaw in the LPM, Ift1 in the diencephalon and heart, Ift2 in the heart, and pitx2 in the LPM at $18 \mathrm{~s}$ or $19-22 \mathrm{~s}$ stages. (TIFF $74 \mathrm{~kb}$ )

Additional file 4: Figure S4. Symmetric charon expression around KV was observed in the majority of klf8 morphants. Representative images of embryos showing stronger charon expression on the right side (A) or left side (B) and symmetric charon expression on both sides (C) of KV are shown. Quantification of different charon expression patterns in embryos injected with different klf8 MOs, control MO or wild type embryo is shown (D). Statistical significance was determined by Student's $t$-test. ${ }^{*} p<0.05$. Error bars indicate standard deviation. (TIFF $397 \mathrm{~kb}$ )

Additional file 5: Figure S5. Expression level of $b m p 2 b$ or $d v r 1$ around tailbud region was not affected by klf8 knockdown. Representative images show similar expression level of bmp2b (A-D) or $d v r 1(\mathbf{E}-\mathbf{H})$ around the tailbud region in the wild type or embryos injected with Klf8-MO1 ${ }^{\text {atg }}$, Klff-MO2 $^{\text {atg }}$ or klf8-4 mm MO1 at $3 \mathrm{~s}$ or bud stages. (TIFF $382 \mathrm{~kb}$ )

\section{Abbreviations}

ASE: Left-side specific enhancers; CRISPR-Cas: Clustered regularly interspaced short palindromic repeats- CRISPR-associated system; CtBP: C-terminal binding protein; DFCs: Dorsal forerunner cells; Hpf: Hours post fertilization; kff: krüppel-like factor 8; KV: Kupffer's vesicle; Ift1: lefty1; Ift2: lefty2; LPM: Lateral plate mesoderm; MO: Morpholino oligomer; Ndr: Nodal-related genes; RT: Room temperature; RT-PCR: Reverse transcription PCR; RT-qPCR: Reverse transcription quantitative real-time PCR; S: Somite

\section{Acknowledgments}

We thank the members of the Core Facility of the Institute of Cellular and Organismic Biology, Academia Sinica, for their assistance with DNA sequencing and confocal imaging. We thank the Taiwan Zebrafish Core Facility at Academia Sinica (TZCAS) for providing the ASAB wild type strain. We thank ZIRC and TZCAS for providing Tg(sox17:gfp $)^{887 /+}$ fish line. We thank Dr. M. Rebagliati for providing full-length spaw cDNA. We thank Ms. Mei-Chen Chen for fish maintenance.

\section{Funding}

This work was supported by Academia Sinica Innovative Translational Agricultural Research Program [542300 to S.P.L.H.] and the Ministry of Science and Technology, Taiwan [MOST 105-2313-B-001-005-MY3 to S.P.L.H.].

\section{Availability of data and materials}

All materials are available by the corresponding author.

\section{Authors' contributions}

CYL, MYT, YCC, YRL, YHL, YFL, HCL, HWL, CHY performed experiments; CYL, MYT, CJH and SPLH analysed data; SPLH conceived the project and wrote the manuscript. All authors read and approved the final manuscript.

\section{Ethics approval and consent to participate}

All animal procedures were approved by the Institutional Animal Care and Use Committee of Academia Sinica (Protocol ID: 15-12-918).

\section{Consent for publication}

Not applicable.

\section{Competing interests}

The authors declare that they have no competing interests.

\section{Publisher's Note}

Springer Nature remains neutral with regard to jurisdictional claims in published maps and institutional affiliations.

\section{Author details}

${ }^{1}$ Department of Bioscience and Biotechnology, National Taiwan Ocean University, Keelung, Taiwan. ${ }^{2}$ Graduate Institute of Life Sciences, National Defence Medical Center, National Defence University, Neihu, Taipei, Taiwan. ${ }^{3}$ Department of Life Science, National Taiwan University, Taipei, Taiwan. ${ }^{4}$ Institute of Cellular and Organismic Biology, Academia Sinica, Nankang, Taipei 11529, Taiwan. ${ }^{5}$ nstitute of Biological Chemistry, Academia Sinica, Taipei, Taiwan. ${ }^{6}$ Present address: Institute of Cellular and Organismic Biology, Academia Sinica, Taipei, Taiwan. 


\section{Received: 5 April 2017 Accepted: 7 July 2017}

\section{Published online: 17 July 2017}

\section{References}

1. Bisgrove BW, Morelli SH, Yost HJ. Genetics of human laterality disorders: insights from vertebrate model systems. Annu Rev Genomics Hum Genet. 2003:4:1-32.

2. Kosaki K, Casey B. Genetics of human left-right axis malformations. Semin Cell Dev Biol. 1998:9:89-99.

3. Hamada H, Meno C, Watanabe D, Saijoh Y. Establishment of vertebrate leftright asymmetry. Nat Rev Genet. 2002;3:103-13.

4. Shiratori $\mathrm{H}, \mathrm{Hamada} H$. The left-right axis in the mouse: from origin to morphology. Development. 2006;133:2095-104.

5. Hirokawa N, Tanaka Y, Okada Y. Left-right determination: involvement of molecular motor KIF3, cilia, and nodal flow. Cold Spring Harb Perspect Biol. 2009;1:a000802.

6. Nonaka S, Tanaka Y, Okada Y, Takeda S, Harada A, Kanai Y, Kido M, Hirokawa N. Randomization of left-right asymmetry due to loss of nodal cilia generating leftward flow of extraembryonic fluid in mice lacking KIF3B motor protein. Cell. 1998:95:829-37.

7. Neugebauer JM, Amack JD, Peterson AG, Bisgrove BW, Yost HJ. FGF signalling during embryo development regulates cilia length in diverse epithelia. Nature. 2009;458:651-4.

8. Thomas J, Morle L, Soulavie F, Laurencon A, Sagnol S, Durand B. Transcriptional control of genes involved in ciliogenesis: a first step in making cilia. Biol Cell. 2010;102:499-513.

9. Nakamura T, Mine N, Nakaguchi E, Mochizuki A, Yamamoto M, Yashiro K, Meno C, Hamada H. Generation of robust left-right asymmetry in the mouse embryo requires a self-enhancement and lateral-inhibition system. Dev Cell. 2006;11:495-504.

10. Schier AF. Nodal signaling in vertebrate development. Annu Rev Cell Dev Biol. 2003;19:589-621.

11. Saijoh Y, Adachi H, Sakuma R, Yeo CY, Yashiro K, Watanabe M, Hashiguchi $H$, Mochida K, Ohishi S, Kawabata M, Miyazono K, Whitman M, Hamada H. Leftright asymmetric expression of lefty 2 and nodal is induced by a signaling pathway that includes the transcription factor FAST2. Mol Cell. 2000;5:35-47.

12. Shiratori $H$, Sakuma $R$, Watanabe M, Hashiguchi $H$, Mochida K, Sakai $Y$, Nishino J, Saijoh Y, Whitman M, Hamada H. Two-step regulation of left-right asymmetric expression of Pitx2: initiation by nodal signaling and maintenance by Nkx2. Mol Cell. 2001;7:137-49.

13. Ryan AK, Blumberg B, Rodriguez-Esteban C, Yonei-Tamura S, Tamura K, Tsukui T, de la Pena J, Sabbagh W, Greenwald J, Choe S, Norris DP, Robertson EJ, Evans RM, Rosenfeld MG, Izpisua Belmonte JC. Pitx2 determines left-right asymmetry of internal organs in vertebrates. Nature. 1998;394:545-51.

14. Essner JJ, Amack JD, Nyholm MK, Harris EB, Yost HJ. Kupffer's vesicle is a ciliated organ of asymmetry in the zebrafish embryo that initiates left-right development of the brain, heart and gut. Development. 2005:132:1247-60.

15. Oteiza $P$, Koppen M, Concha ML, Heisenberg CP. Origin and shaping of the laterality organ in zebrafish. Development. 2008;135:2807-13.

16. Amack JD. Salient features of the ciliated organ of asymmetry. BioArchitecture. 2014;4:6-15.

17. Wang G, Cadwallader AB, Jang DS, Tsang M, Yost HJ, Amack JD. The Rho kinase Rock2b establishes anteroposterior asymmetry of the ciliated Kupffer's vesicle in zebrafish. Development. 2011;138:45-54.

18. Smith DJ, Montenegro-Johnson TD, Lopes SS. Organized chaos in Kupffer's vesicle: how a heterogeneous structure achieves consistent left-right patterning. BioArchitecture. 2014;4:119-25.

19. Bisgrove BW, Snarr BS, Emrazian A, Yost HJ. Polaris and Polycystin-2 in dorsal forerunner cells and Kupffer's vesicle are required for specification of the zebrafish left-right axis. Dev Biol. 2005;287:274-88.

20. Borovina A, Superina S, Voskas D, Ciruna B. Vangl2 directs the posterior tilting and asymmetric localization of motile primary cilia. Nat Cell Biol. 2010;12:407-12.

21. Wang $X$, Yost HJ. Initiation and propagation of posterior to anterior (PA) waves in zebrafish left-right development. Dev Dyn. 2008;237:3640-7.

22. Long S, Ahmad N, Rebagliati M. The zebrafish nodal-related gene southpaw is required for visceral and diencephalic left-right asymmetry. Development. 2003;130:2303-16.

23. Ahmad N, Long S, Rebagliati M. A southpaw joins the roster: the role of the zebrafish nodal-related gene southpaw in cardiac LR asymmetry. Trends Cardiovasc Med. 2004;14:43-9.
24. Hashimoto H, Rebagliati M, Ahmad N, Muraoka O, Kurokawa T, Hibi M Suzuki T. The Cerberus/Dan-family protein Charon is a negative regulator of Nodal signaling during left-right patterning in zebrafish. Development. 2004 131:1741-53.

25. Chocron S, Verhoeven MC, Rentzsch F, Hammerschmidt M, Bakkers J. Zebrafish Bmp4 regulates left-right asymmetry at two distinct developmental time points. Dev Biol. 2007;305:577-88.

26. Smith KA, Noel E, Thurlings I, Rehmann H, Chocron S, Bakkers J. Bmp and nodal independently regulate lefty 1 expression to maintain unilateral nodal activity during left-right axis specification in zebrafish. PLoS Genet. 2011;7: e1002289.

27. Lenhart KF, Lin SY, Titus TA, Postlethwait JH, Burdine RD. Two additional midline barriers function with midline lefty 1 expression to maintain asymmetric Nodal signaling during left-right axis specification in zebrafish. Development. 2011;138:4405-10.

28. Kaczynski J, Cook T, Urrutia R. Sp1- and Kruppel-like transcription factors. Genome Biol. 2003;4:206

29. Pearson R, Fleetwood J, Eaton S, Crossley M, Bao S. Kruppel-like transcription factors: a functional family. Int J Biochem Cell Biol. 2008;40:1996-2001.

30. Lahiri SK, Zhao J. Kruppel-like factor 8 emerges as an important regulator of cancer. Am J Transl Res. 2012;4:357-63.

31. van Vliet J, Turner J, Crossley M. Human Kruppel-like factor 8: a CACCC-box binding protein that associates with CtBP and represses transcription. Nucleic Acids Res. 2000;28:1955-62

32. Zhang P, Basu P, Redmond LC, Morris PE, Rupon JW, Ginder GD, Lloyd JA. A functional screen for Kruppel-like factors that regulate the human gammaglobin gene through the CACCC promoter element. Blood Cells Mol Dis. 2005:35:227-35

33. Funnell AP, Mak KS, Twine NA, Pelka GJ, Norton LJ, Radziewic T, Power M, Wilkins MR, Bell-Anderson KS, Fraser ST, Perkins AC, Tam PP, Pearson RC, Crossley M. Generation of mice deficient in both KLF3/BKLF and KLF8 reveals a genetic interaction and a role for these factors in embryonic globin gene silencing. Mol Cell Biol. 2013;33:2976-87.

34. Zhao J, Bian ZC, Yee K, Chen BPC, Chien S, Guan J-L. Identification of transcription factor KLF8 as a downstream target of focal adhesion kinase in its regulation of cyclin D1 and cell cycle progression. Mol Cell. 2003;11:1503-15.

35. Tsai MY, Lu YF, Liu YH, Lien HW, Huang CJ, Wu JL, Hwang SP. Modulation of p53 and met expression by Kruppel-like factor 8 regulates zebrafish cerebellar development. Dev Neurobiol. 2015;75:908-26.

36. Kimmel CB, Ballard WW, Kimmel SR, Ullmann B, Schilling TF. Stages of embryonic development of the zebrafish. Dev Dyn. 1995;203:253-310.

37. Chen J, Ruan H, Ng SM, Gao C, Soo HM, Wu W, Zhang Z, Wen Z, Lane DP, Peng J. Loss of function of def selectively up-regulates Delta113p53 expression to arrest expansion growth of digestive organs in zebrafish. Genes Dev. 2005:19:2900-11.

38. Jao LE, Wente SR, Chen W. Efficient multiplex biallelic zebrafish genome editing using a CRISPR nuclease system. Proc Natl Acad Sci U S A. 2013;110: 13904-9.

39. Bustin SA, Benes V, Garson JA, Hellemans J, Huggett J, Kubista M, Mueller R, Nolan T, Pfaffl MW, Shipley GL, Vandesompele J, Wittwer CT. The MIQE guidelines: minimum information for publication of quantitative real-time PCR experiments. Clin Chem. 2009;55:611-22.

40. Sampaio P, Ferreira RR, Guerrero A, Pintado P, Tavares B, Amaro J, Smith AA, Montenegro-Johnson T, Smith DJ, Lopes SS. Left-right organizer flow dynamics: how much cilia activity reliably yields laterality? Dev Cell. 2014;29: 716-28.

41. McConnell BB, Yang W. Mammalian Kruppel-like factors in health and diseases. Physiol Rev. 2010;90:1337-81.

42. Noel ES, Verhoeven M, Lagendijk AK, Tessadori F, Smith K, Choorapoikayil S, den Hertog J, Bakkers J. A Nodal-independent and tissue-intrinsic mechanism controls heart-looping chirality. Nat Commun. 2013;4:2754.

43. Matsui T, Ishikawa $H$, Bessho Y. Cell collectivity regulation within migrating cell cluster during Kupffer's vesicle formation in zebrafish. Front Cell Dev Biol. 2015:3:27.

44. Amack JD, Wang X, Yost HJ. Two T-box genes play independent and cooperative roles to regulate morphogenesis of ciliated Kupffer's vesicle in zebrafish. Dev Biol. 2007;310:196-210.

45. Oteiza P, Koppen M, Krieg M, Pulgar E, Farias C, Melo C, Preibisch S, Muller D, Tada M, Hartel S, Heisenberg CP, Concha ML. Planar cell polarity signalling regulates cell adhesion properties in progenitors of the zebrafish laterality organ. Development. 2010;137:3459-68. 
46. Matsui $T$, Thitamadee $S$, Murata $T$, Kakinuma $H$, Nabetani $T$, Hirabayashi $Y$, Hirate Y, Okamoto H, Bessho Y. Canopy1, a positive feedback regulator of FGF signaling, controls progenitor cell clustering during Kupffer's vesicle organogenesis. Proc Natl Acad Sci U S A. 2011;108:9881-6.

47. Wang G, Manning ML, Amack JD. Regional cell shape changes control form and function of Kupffer's vesicle in the zebrafish embryo. Dev Biol. 2012;370:52-62.

48. Schlange T, Arnold HH, Brand T. BMP2 is a positive regulator of Nodal signaling during left-right axis formation in the chicken embryo. Development. 2002;129:3421-9.

49. Mine N, Anderson RM, Klingensmith J. BMP antagonism is required in both the node and lateral plate mesoderm for mammalian left-right axis establishment. Development. 2008;135:2425-34.

50. Fujiwara T, Dehart DB, Sulik KK, Hogan BL. Distinct requirements for extraembryonic and embryonic bone morphogenetic protein 4 in the formation of the node and primitive streak and coordination of left-right asymmetry in the mouse. Development. 2002;129:4685-96.

51. Raya A, Izpisua Belmonte JC. Unveiling the establishment of left-right asymmetry in the chick embryo. Mech Dev. 2004;121:1043-54.

52. Peterson AG, Wang $X$, Yost HJ. Dvr1 transfers left-right asymmetric signals from Kupffer's vesicle to lateral plate mesoderm in zebrafish. Dev Biol. 2013; 382:198-208.

53. Kok FO, Shin M, Ni CW, Gupta A, Grosse AS, van Impel A, Kirchmaier BC, Peterson-Maduro J, Kourkoulis G, Male I, DeSantis DF, Sheppard-Tindell S, Ebarasi L, Betsholtz C, Schulte-Merker S, Wolfe SA, Lawson ND. Reverse genetic screening reveals poor correlation between morpholino-induced and mutant phenotypes in zebrafish. Dev Cell. 2015:32:97-108.

54. Rossi A, Kontarakis Z, Gerri C, Nolte H, Holper S, Kruger M, Stainier DY. Genetic compensation induced by deleterious mutations but not gene knockdowns. Nature. 2015;524:230-3.

55. Spicer OS, Wong TT, Zmora N, Zohar Y. Targeted mutagenesis of the hypophysiotropic Gnrh3 in Zebrafish (Danio rerio) reveals no effects on reproductive performance. PLoS One. 2016;11:e0158141.

56. Lin MJ, Lee SJ. Stathmin-like 4 is critical for the maintenance of neural progenitor cells in dorsal midbrain of zebrafish larvae. Sci Rep. 2016;6:36188.

\section{Submit your next manuscript to BioMed Central and we will help you at every step:}

- We accept pre-submission inquiries

- Our selector tool helps you to find the most relevant journal

- We provide round the clock customer support

- Convenient online submission

- Thorough peer review

- Inclusion in PubMed and all major indexing services

- Maximum visibility for your research

Submit your manuscript at www.biomedcentral.com/submit 